\title{
Nonlinear Dynamics of an Ambient Noise Driven Array of Coupled Graphene Nanostructured Devices for Energy Harvesting
}

\author{
A. El Aroudi ${ }^{1, a}$, M. López-Suárez ${ }^{2}$, E. Alarcón ${ }^{3}$, R. Rurali ${ }^{4}$, and G. Abadal ${ }^{2}$ \\ 1 Department of Electronics, Electrical Engineering and Automatic Control, Universitat Rovira i Virgili, Tarragona, Spain \\ 2 Universitat Autònoma de Barcelona, Spain \\ 3 Universitat Politècnica de Catalunya \\ 4 Institut de Ciència de Materials de Barcelona (ICMAB-CSIC), Campus de Bellaterra, 08193, Barcelona, Spain
}

\begin{abstract}
Nonlinearities have been shown to play an important role in increasing the extracted energy of energy harvesting devices at the macro and micro scales. Vibration-based energy harvesting on the nano scale has also received attention. In this paper, we characterize the nonlinear dynamical behavior of an array of three coupled strained nanostructured graphene for its potential use in energy harvesting applications. The array is formed by three compressed vibrating membrane graphene sheet subject to external vibrational noise excitation. We present the continuous time dynamical model of the system in the form of a double-well three degree of freedom system. Random vibrations are considered as the main ambient energy source for the system and its performances in terms of the probability density function, RMS or amplitude value of the position, FFT spectra and state plane trajectories are presented in the steady state non-equilibrium regime when the noise level is considered as a control parameter.
\end{abstract}

\section{Introduction}

Energy harvesting can be performed both at the macro [1], micro [3] and even at nano scales [2]. Unfortunately, most of the available power sources have very low frequency oscillations while the resonant frequency of any nano-mechanica device is paradoxically high ( $\propto \mathrm{GHz}$ ) which make many issues related to their performances as energy harvesters yet to be solved, particularly that of extracting energy at low frequencies. Nonlinearities in energy harvesting nano devices may play a vital role to solve these issues. Recent works have used nano devices such as piezoelectric Zinc Oxide ( $\mathrm{ZnO}$ ) nanowires [4] where mechanical energy coming from light wind and body movement was converted to electrical energy by a coupled piezoelectric-semiconductor process. Graphene is a newly discovered nano material that has interesting peculiar electronic and mechanical properties making it a promising candidate for future electronic applications [5]. In [6] it has been shown that a strained nanostructured graphene posses an intrinsic mechanical nonlinearity and it can be used for nonlinear energy harvesting at the nanoscale. There, it has been also shown that that when weakly compressed, the graphene sheet behaves like a double-well system and that for a critical value of the compression ratio, the harvested power is optimal.

The aim of this paper is to study the nonlinear dynamic behavior of such a device for possible use in energy harvesting applications. To accomplish this aim, the rest of the paper is organized as follows. In Section II, a brief description of the mathematical model of the graphene harvester is provided. Section III presents the dynamical behavior of the device in the absence of any external excitation. Equilibrium points and their stability are studied in the same

\footnotetext{
a e-mail: abdelali.elaroudi@urv.cat
}

section. In Section IV, the performances of the system under the random excitation are studied in terms of RMS levels. Finally, conclusions are provided in the last section.

\section{Mathematical model of the graphene vibrating membrane for energy harvesting applications}

\subsection{Nonlinear mathematical model}

We consider three flat suspended graphene sheets with a compression ratio defined as $\varepsilon=\left(L_{0}-L\right) / L_{0}$, where $L$ is the length of each graphene sheet and $L_{0}$ their equilibrium value in absence of any compression (Figure 1). Let us consider different values of the compression ratio $\varepsilon$ between $0 \%$ (uncompressed graphene) and $10 \%$.

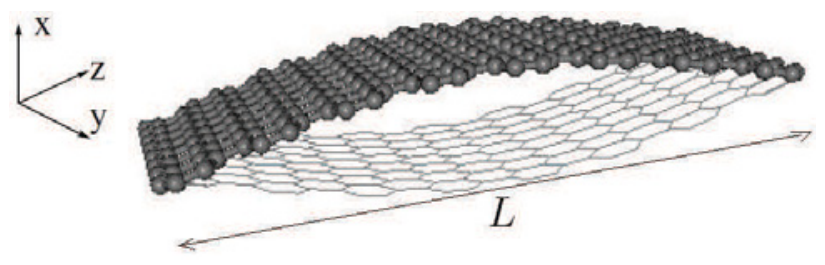

Fig. 1. Uncompressed or weakly compressed graphene membrane [6].

Let $\xi(t)=\sigma^{2} \eta(t)$, where $\eta(t)$ is a white noise excitation with a Gaussian distribution noise whose mean value is zero, its variance is $\sigma^{2}$. By developing the Euler-Bernoulli equation for clamped beams, the following mass-spring 
Table 1. Coefficients $v_{i}(i=2,4,6,8)$ for the potential energy for different values of the compression ratio $\varepsilon$.

\begin{tabular}{|c|c|c|c|c|}
\hline$\varepsilon$ & $v_{8} / 56$ & $v_{6} / 30$ & $v_{4} / 12$ & $v_{2} / 2$ \\
\hline 0 & $6.57 \cdot 10^{50}$ & $-5.73 \cdot 10^{33}$ & $6.74 \cdot 10^{16}$ & 0.0096 \\
\hline 0.025 & $1.30 \cdot 10^{51}$ & $-1.36 \cdot 10^{34}$ & $9.26 \cdot 10^{16}$ & -0.036 \\
\hline 0.05 & $2.56 \cdot 10^{51}$ & $-2.29 \cdot 10^{34}$ & $1.14 \cdot 10^{17}$ & -0.078 \\
\hline 0.075 & $2.76 \cdot 10^{51}$ & $-2.34 \cdot 10^{34}$ & $1.16 \cdot 10^{17}$ & -0.112 \\
\hline 0.1 & $3.89 \cdot 10^{51}$ & $-3.43 \cdot 10^{34}$ & $1.49 \cdot 10^{17}$ & -0.165 \\
\hline
\end{tabular}

dynamic model is obtained for the considered coupled graphene membranes [6]

$$
\begin{aligned}
& m \ddot{x}_{1}=-\left.\frac{\partial V(x)}{\partial x}\right|_{x=x_{1}}-\left.\frac{\partial V(x)}{\partial x}\right|_{x=x_{2}-x_{1}}-b \dot{x}_{1}+\sigma^{2} \eta(t)(1) \\
& m \ddot{x}_{2}=-\left.\frac{\partial V(x)}{\partial x}\right|_{x=x_{2}-x_{3}}-b \dot{x}_{2}+\left.\frac{\partial V(x)}{\partial x}\right|_{x=x_{1}-x_{2}}-b \dot{x}_{2}(2) \\
& m \ddot{x}_{3}=-\left.\frac{\partial V(x)}{\partial x}\right|_{x=x_{3}-x_{4}}-b \dot{x}_{3}+\left.\frac{\partial V(x)}{\partial x}\right|_{x=x_{2}-x_{3}}-b \dot{x}_{2}(3) \\
& m \ddot{x}_{4}=-\left.\frac{\partial V(x)}{\partial x}\right|_{x=x_{4}}-b \dot{x}_{4}+\left.\frac{\partial V(x)}{\partial x}\right|_{x=x_{3}-x_{4}}-b \dot{x}_{2}
\end{aligned}
$$

where $x_{i}, i=1,2,3$ are the displacements for the coupled graphene membranes, $m$ represents the effective mass of each of the layers and $b$ stands for their damping factor. It should be noted that for simplicity it is assumed that all the membranes are identic, i.e, they have the same mass and the same damping coefficient. The potential energy is given by [6]

$$
V(x)=\frac{1}{56} v_{8} x^{8}+\frac{1}{30} v_{6} x^{6}+\frac{1}{12} v_{4} x^{4}+\frac{1}{2} v_{2} x^{2}
$$

The coefficients $v_{i}(i=2,4,6,8)$ are given in Table 1 for different values of compression coefficient $\varepsilon$. These values have been obtained by means of ab initio density-functional calculations with the SIESTA code [6], [7] where the graphene sheet is modeled at a fully atomistic level and its electronic structure solved quantum mechanically. Figure 2 shows the plot of $V(x)$ in terms of the position $x$.

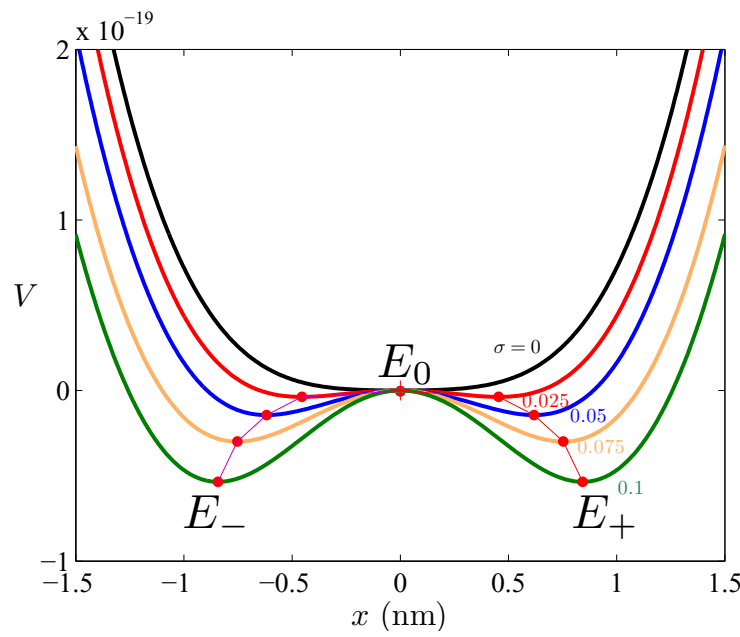

Fig. 2. Potential $V(x)$ for different values of the compression factor $\varepsilon$. For $\varepsilon \neq 0$, equilibrium points are also plotted. $E_{+}$and $E_{-}$ are attractors while $E_{0}$ is a saddle point. The

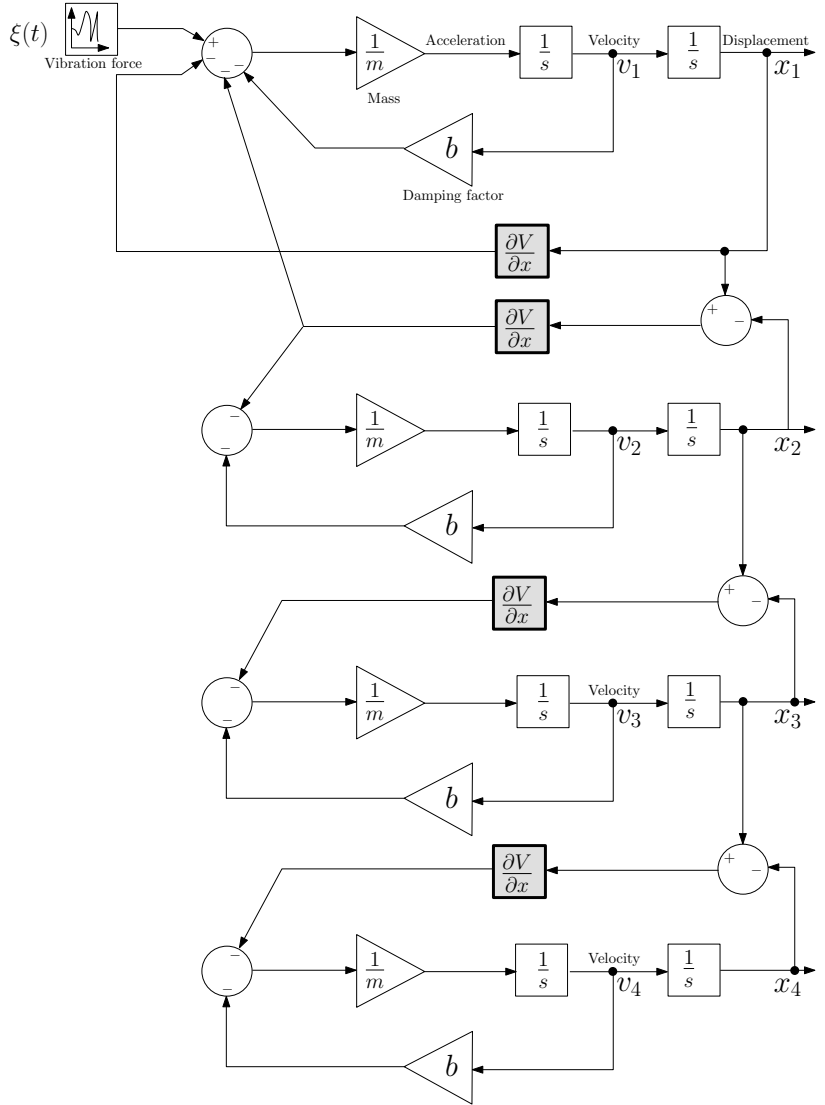

Fig. 3. Simulink block diagram of four coupled graphene membranes.

\section{Dynamical behavior from numerical simulations}

\subsection{Dynamics of a single graphene membrane}

The nonlinear dynamics of a single graphene membrane has been studied in [6] and [8]. We resume here the results for comparison with the coupled graphene membranes. Let also consider a compressed graphene with compression coefficient $\varepsilon=0.1$ in such a way that the origin is a saddle point (non stable). The nonlinear model of the system is numerically solved. A Matlab-simulink model was built for the system equation modeling the graphene nano harvester device according to its mathematical model (Fig. 3). A random excitation with a normal distribution and a maximum bandwidth $\omega_{\mathrm{bw}}$ is considered as a main input of the system.

When such noise is applied to the system, the probability to swing between the two equilibrium point increases. Consequently noise can be used to increase the RMS value of the displacement and accordingly to raise the harvested energy. Let us also consider a compressed graphene sheet with compression coefficient $\varepsilon \neq 0$ in such a way that the origin is a saddle point (non stable). For some specific values of noise level, the system was simulated during $500 \mathrm{~ns}$ and the time-series, phase space, probability density, and amplitude spectra are plotted. Figures 4-5 show the dynamics of the system for different values of noise level. The following particular cases are plotted in these figures

- $\sigma^{2}=1 \mathrm{pN}$, the noise intensity is weak and the system evolves to the vicinity of one of the stable equilibrium 


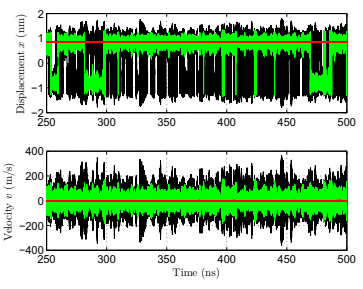

(a) Time series.

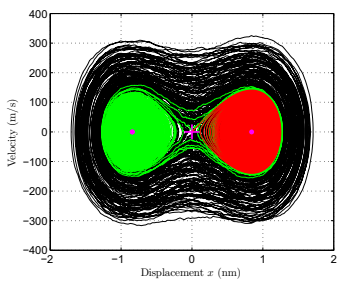

(b) Phase plane $(x, v)$.

Fig. 4. Dynamics of the graphene membrane under a random excitation $\sigma^{2}=1 \mathrm{pN}\left(\right.$ red), $\sigma^{2}=50.5 \mathrm{pN}$ (green) and $\sigma^{2}=100 \mathrm{pN}$ (black).

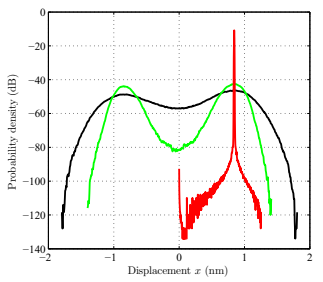

(a) Probability density function of $x$.

Fig. 5. Spectrum and probability density function corresponding to Fig. 4. Note that the spectrum is spread down to low frequency regions when the noise intensity is sufficiently large so that the potential barrier can be crossed.

points $X_{-}$or $X_{+}$depending on the initial conditions. The probability to swing between the two equilibria is almost zero. The vicinities of $X_{-}$and $X_{+}$correspond to the unique set with non-zero values of the probability density function. The energy of the system is concentrated in a limited range of frequency near the resonant frequency $(\approx 50 \mathrm{GHz})$.

$-\sigma^{2}=50.5 \mathrm{pN}$, the noise intensity is sufficient to make the system to leave the vicinity of the stable equilibrium points $X_{-}$or $X_{+}$which makes the system to swing between them. The range within which the displacement includes the interval $\left(X_{-}, X_{+}\right)$. The equilibria $X_{-}$ and $X_{+}$correspond to the maximum of the probability density function.

$-\sigma^{2}=100 \mathrm{pN}$, the noise intensity is in such a way that the probability of swinging between the stable equilibrium points $X_{-}$or $X_{+}$is larger and the system presents larger velocities and displacements and therefore the harvested energy will also be larger.

\subsection{Dynamics of the coupled membranes}

In this section, only two values of noise intensity are considered, namely $\sigma^{2}=1 \mathrm{pN}$ and $\sigma^{2}=50 \mathrm{pN}$, and different diagrams in the form of times series, state planes, FFT spectra and probability density function of the displacement are obtained as for the single membrane case.

Figure 6 shows the time domain waveforms of the system. For low values of noise intensity the system stays in the vicinity of the equilibrium point. For low values of noise intensity the system stays in the vicinity of the equilibrium point.
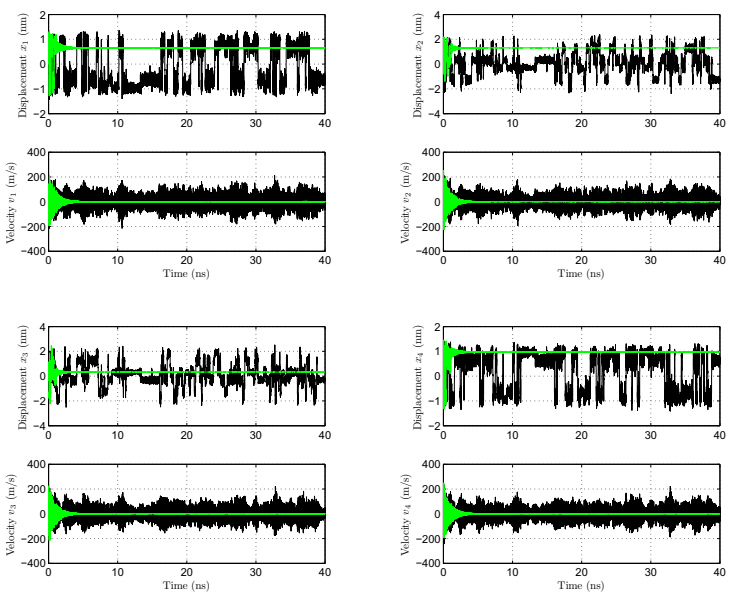

Fig. 6. Time domain waveforms of the displacements $x_{i}$ and velocities $v_{i},(i=1 . .4)$ corresponding to the four graphene membranes for two values of noise intensities.
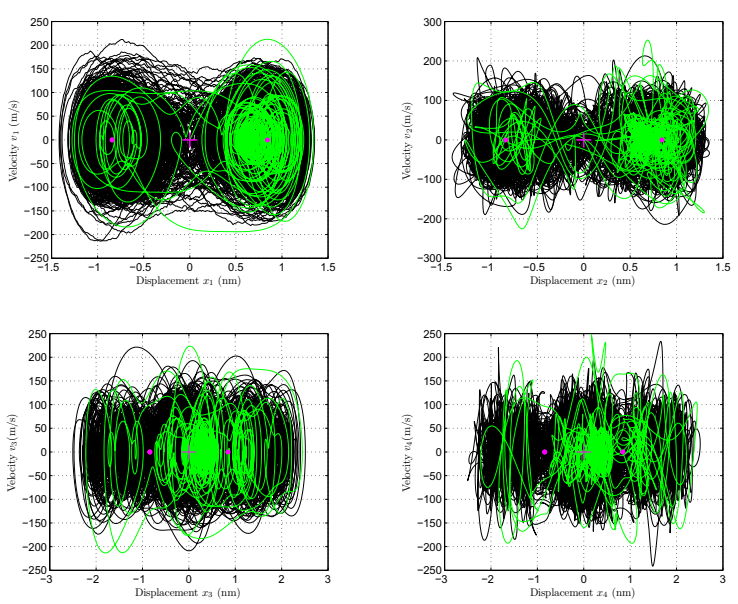

Fig. 7. The state space in the planes $\left(x_{i}, v_{i}\right),(i=1 . .4)$ corresponding to the four graphene membranes for two values of noise intensities.

Figure 7 shows the projection of the state plane time of the system in the planes $\left(x_{i}, v_{i}\right),(i=1 . .4)$ corresponding to each graphene membrane. For relatively high values of noise intensity, the trajectory can only evolve to an equilibrium point being the ambient vibration in this case not sufficient to make the system to oscillate between the two regions. When the noise intensity increases, as in the case of the single membrane, the system trajectory visits more the different equilibrium points and larger oscillation amplitude can be reached. All the four membranes are characterized by the same dynamics.

Figure 8 depicts the FFT spectra of the displacement corresponding to each graphene membrane. While a clear single resonant frequency can be observed for the low intensity noise case, the spectrum corresponding to a relatively high value of noise intensity is spread a broad range of frequencies. This occurs for all the membranes.

Figure 9 shows the probability density functions calculated for the different displacements of the membranes. For low values of noise intensity the probability of finding the system in the vicinity of one of its equilibrium points is 

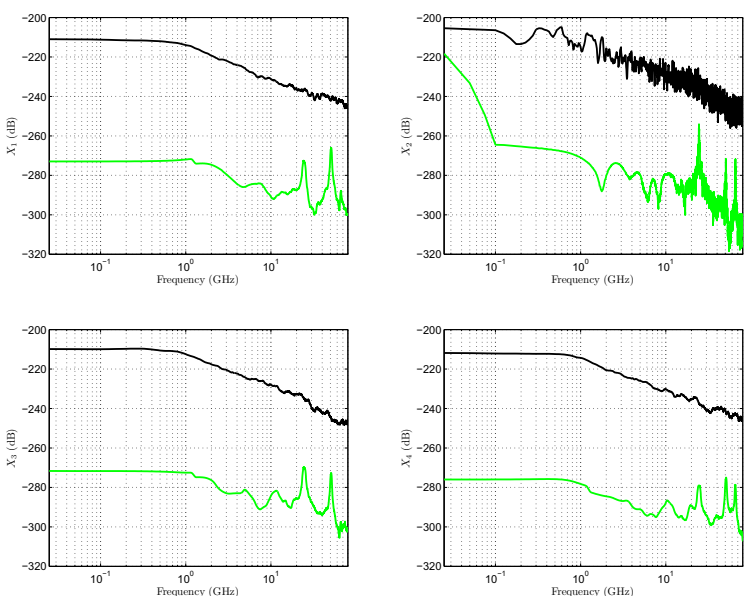

Fig. 8. FFT spectra of the displacements $x_{i}(i=1 . .4)$ corresponding to the four graphene membranes for two values of noise intensities.
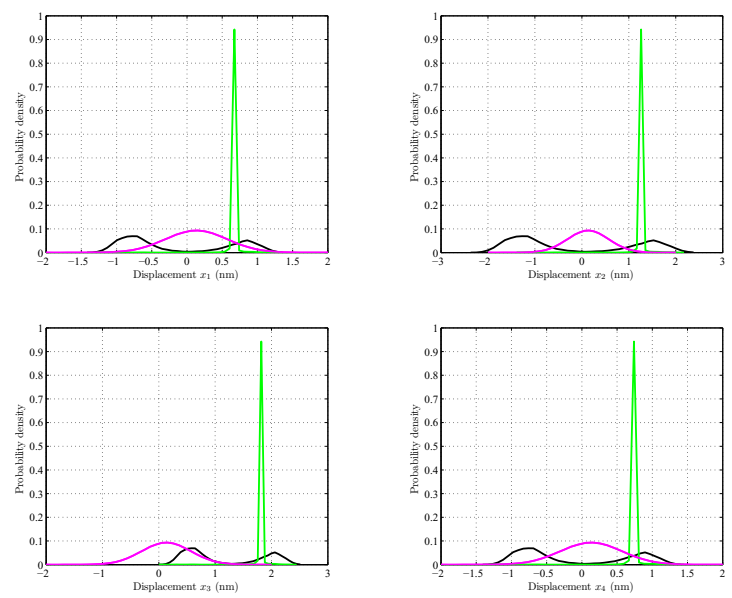

Fig. 9. Probability density function of the displacements $x_{i}(i=$ 1..4) for two values of noise intensities. For reference, the probability density of the noise driving signal is also plotted

practically one. For relatively high values of noise intensity the probability to visit a wider region increases being the maximum probability that corresponding to the vicinity of the equilibrium points.

\section{Conclusions}

In this paper we have considered the nonlinear dynamics of four identical coupled nanostructured graphene vibrating membranes for ambient energy applications at the nanoscale. The compressed graphen present a nonlinearity that has been shown to play an important role in increasing the efficiency of this energy harvesting device by increasing the RMS values of the displacement and the velocity. We presented a continuous-time nonlinear dynamical model of the coupled system. When random vibrations are considered as the main ambient energy source for the system, the performances of the system as an energy harvester are presented in the steady state non-equilibrium regime when the noise level for a certain value of the compression ratio.

\section{Acknowledgments}

This work was partially supported by the Ministerio de Ciencia e Innovación under grant CSD2009-00046.

\section{References}

1. L. Gammaitoni, H. Vocca, I. Neri, F. Travasso and F. Orfei, "Vibration Energy Harvestng: Linear and Nonlinear Oscillator Approaches," in Sustainable Energy Harvesting Technologies-Past, Present and Future, pp. 171-190, 2007.

2. Igor Neri, Flavio Travasso, Helios Vocca, Luca Gammaitoni, "Nonlinear noise harvesters for nanosensors," Nano Communication Networks vol. 2, no. 4, 2011, pp. 230-234.

3. Huicong Liu; Cho Jui Tay; Chenggen Quan; Kobayashi, T.; Chengkuo Lee; "Piezoelectric MEMS Energy Harvester for Low-Frequency Vibrations With Wideband Operation Range and Steadily Increased Output Power," Journal of Microelectromechanical Systems, vol.20, no.5, pp.1131-1142, Oct. 2011.

4. Sheng $\mathrm{Xu}$, Yong Qin, Chen $\mathrm{Xu}$, Yaguang Wei, Rusen Yang and Zhong Lin Wang "Self-powered nanowire devices," Nature nanotechnology, vol. 5, no. 5, 2010.

5. S. A. Imam, S. Sabri and T. Szkopek, "Low-frequency noise and hysteresis in graphene field-effect transistors on oxide S.A.," Micro and Nano Letters, IET, vol.5, no.1, pp.37-41, Feb. 2010.

6. M. López-Suárez, R. Rurali, L. Gammaitoni and G. Abadal "Nanostructured Graphene for Energy Harvesting," Physical Rweview B, vo. 84, No. 161401, pp.1-5, 2011.

7. J. M. Soler, E. Artacho, J. D. Gale, A. García, J. Junquera, P. Ordejon, and D. Sánchez-Portal, "The SIESTA method for ab initio order-N materials simulation," J. Phys. Condens. Matter vol. 14, no. 11, pp. 2745, 2002.

8. A. El Aroudi, M. Lopez-Suarez, R. Rurali, E. Alarcon and G. Abadal "Nonlinear Dynamics in a Nanostructured Graphene Device for Energy Harvesting Applications", IEEE International Symposium on Circuits and Systems, 2013, ISCAS 2013, May 2013, pp. $2727-$ 2730, Beijing, China. 